\title{
Search for gamma-ray emission from electromagnetic counterparts of gravitational wave sources with the CALET calorimeter
}

\author{
Masaki Mori \\ Department of Physical Sciences, Ritsumeikan University, Shiga 525-8577, Japan \\ E-mail: morimefc.ritsumei.ac.jp \\ Yoichi Asaoka \\ Research Institute for Science and Engineering, Waseda University, Tokyo 169-8555, Japan \\ E-mail: yoichi.asaoka@aoni.waseda.jp \\ for the CALET collaboration
}

\begin{abstract}
The first detection of a gravitational wave event by advanced LIGO in 2015 prompted the search for and study of electromagnetic counterparts to gravitational wave events on a worldwide scale. If an electromagnetic signal is also observed, it would provide additional information that can give us significantly better constraints on the parameters (such as mass, orbit, spin) of the binary system. The CALorimetric Electron Telescope (CALET) is a wide field-of-view instrument to study high-energy cosmic-rays onboard the International Space Station accumulating scientific data since October 2015. Gamma-rays in the energy range of $1 \mathrm{GeV}$ to $10 \mathrm{TeV}$ are monitored continuously with its 30 radiation-length deep calorimeter (CAL), with a field-of-view of about 2 steradian and an angular resolution better than 0.4 degree above $10 \mathrm{GeV}$. We have already reported an upper limit on the GW151226 event claimed by advanced LIGO [1]. In this paper, we describe the refined data analysis of CAL for the GW151226 event and the preliminary results on the third gravitational event, GW170104. Hard X-ray results from CALET Gamma-ray Burst Monitor will be presented separately.
\end{abstract}

35th International Cosmic Ray Conference -ICRC2017-

12-20 July, 2017

Bexco, Busan, Korea 


\section{Introduction}

\subsection{Gravitational events}

The first gravitational-wave detection by the Laser Interferometer Gravitational-Wave Observatory (LIGO), dubbed GW150914, confirmed the existence not only of gravitational waves from astronomical objects but also of a binary black hole system with several tens of solar masses [2]. Based solely on the gravitational-wave signals recorded by two LIGO detectors, the current hypothesis is that GW150914 was the result of a merger of two black holes with initial masses of $36_{-4}^{+5} M_{\odot}$ and $29_{-4}^{+4} M_{\odot}$ at the luminosity distance of $410_{-180}^{+160} \mathrm{Mpc}$. The Fermi Gamma-ray Burst Monitor (Fermi-GBM) reported a possible weak gamma-ray transient source above $50 \mathrm{keV}$ at $0.4 \mathrm{~s}$ after the GW150914 trigger [3]. However, the upper limit provided by the INTEGRAL ACS instrument in a gamma-ray energy band similar to the Fermi-GBM energy band is not consistent with a possible gamma-ray counterpart of GW150914 suggested by the Fermi-GBM [4]. No electromagnetic counterpart of GW150914 was found in radio, optical, near-infrared, X-ray and high-energy gamma-ray [5].

GW151226 (LIGO-Virgo trigger ID: G211117) is the second gravitational-wave candidate identified by both LIGO Hanford Observatory and LIGO Livingston Observatory with a high significance (the false-alarm rate of less than one per 1000 years by the on-line search) at 03:38:53.647 UT on 2015 December 26 [6. The event is very likely a binary black hole merger with initial black hole masses of $14.2_{-3.7}^{+8.3} M_{\odot}$ and $7.5_{-2.3}^{+2.3} M_{\odot}$ and a final black hole mass of $20.8_{-1.7}^{+6.1} M_{\odot}$ [ $[7]$. The luminosity distance of the source is estimated as $440_{-190}^{+180} \mathrm{Mpc}$, which corresponds to a redshift of $0.09_{-0.04}^{+0.03}$. As far as the electromagnetic counterpart search of GW151226 in the gamma-ray regime is concerned, Fermi-GBM [8], Fermi Large Area Telescope (LAT) «8], High-Altitude Water Cherenkov Observatory [9], and Astrosat-CZTI [10] reported no detections around the GW trigger time. According to Racusin et al. [8], the flux upper limit of Fermi-GBM is from $4.5 \times 10^{-7}$ to $9 \times 10^{-7} \mathrm{erg} \mathrm{cm}^{-2} \mathrm{~s}^{-1}$ in the $10-1000 \mathrm{keV}$ band. The Fermi-LAT flux upper limit using the first orbit data after the LIGO trigger is from $2.6 \times 10^{-10}$ to $7.8 \times 10^{-9} \mathrm{erg} \mathrm{cm}^{-2} \mathrm{~s}^{-1}$ in the $0.1-1 \mathrm{GeV}$ band.

Recently the third candidate, GW170104 (LIGO-Virgo trigger ID: G268556), was identified similarly by the LIGO-Virgo collaboration at 10:11:58.6 UTC on 2017 January 2017 [11]. The inferred component black hole masses are $31.2_{-6.0}^{+8.4} M_{\odot}$ and $19.4_{-5.9}^{+5.3} M_{\odot}$, and a final black hole mass of $48.7_{-4.6}^{+5.7} M_{\odot}$. The luminosity distance of the source is estimated as $880_{-390}^{+450} \mathrm{Mpc}$, which corresponds to a redshift of $0.18_{-0.07}^{+0.08}$. Some electromagnetic counterpart searches at high energies were reported: Astrosat-CZTI reported no detection of a hard X-ray signal [12]. MAXI/GSC reported upper limits in the $2-20 \mathrm{keV}$ band [13]. AGILE reported non-detection of a gamma-ray counterpart around the detection time in the $50 \mathrm{MeV}-30 \mathrm{GeV}$ band, but a weak event lasting about $32 \mathrm{~ms}$ and occurring $0.46 \pm 0.05 \mathrm{~s}$ before $T_{0}$ was found in the omni-directional MCAL data in the $0.4-100 \mathrm{MeV}$ band [14].

\subsection{CALET observation}

The CALorimetric Electron Telescope (CALET) mission [15], which was successfully launched and emplaced on the Japanese Experiment Module-Exposed Facility of the International Space 
Station (ISS) in 2015 August, was fully operational at the LIGO trigger times of GW151226 and GW170104.

CALET consists of two scientific instruments. The Calorimeter (CAL) is the main instrument, which is capable of observing high-energy electrons from $\sim 1 \mathrm{GeV}$ to $\sim 20 \mathrm{TeV}$, protons, helium, and heavy nuclei from $\sim 10 \mathrm{GeV}$ to $1000 \mathrm{TeV}$ and gamma-rays from $\sim 1 \mathrm{GeV}$ to $\sim 10 \mathrm{TeV}$. The field of view (FOV) of CAL is $\sim 45^{\circ}$ from the zenith direction. The energy resolution and the angular resolution for gamma rays are estimated as $3 \%$ and $0.4^{\circ}$, respectively, at $10 \mathrm{GeV}$ [16, 17]. It consists of three main components: the CHarge Detector (CHD), the IMaging Calorimeter (IMC), and the Total AbSorption Calorimeter (TASC) (Fig 1). CHD is made of a set of X- and Y-direction array of 14 plastic scintillator strips $(32 \mathrm{~mm} \times 10 \mathrm{~mm} \times 450 \mathrm{~mm})$, IMC is composed of 8 layers of $\mathrm{X}$ - and Y-direction array of 448 scintillation fibers ( $\mathrm{SciFi}, 1 \mathrm{~mm} \times 1 \mathrm{~mm} \times$ $448 \mathrm{~mm}$ ) separated by tungsten plate with a total of

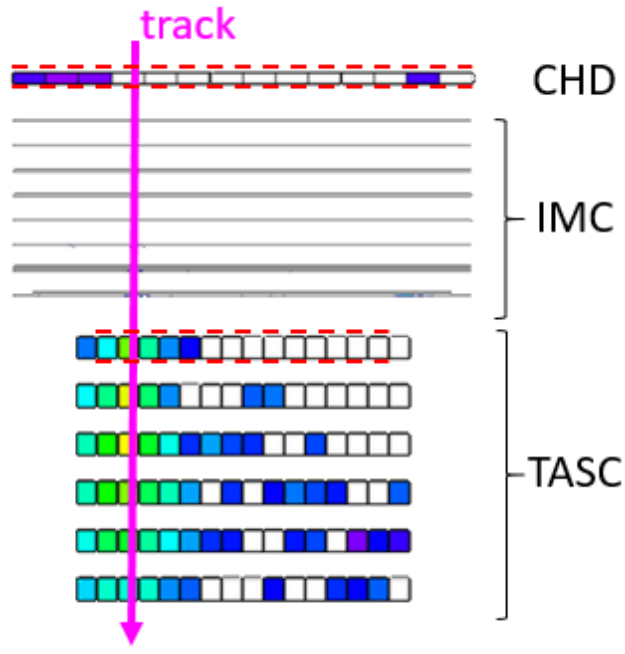

Figure 1: Schematic cross-sectional view of CAL with a sample event. We require that a track should cross the CHD (full area) and the TASC-top (with at least a $2 \mathrm{~cm}$ margin from the outer edge as shown by dashed lines).

3 radiation lengths $\left(X_{0}\right)$ thickness, and TASC is made of 6 layers of X-and Y-array of 16 PWO scintillation crystals $(19 \mathrm{~mm} \times 20 \mathrm{~mm} \times 326 \mathrm{~mm})$ in total of $27 X_{0}$ thickness (see ref. [18] for detail). The CAL gamma-ray performance and initial CAL gamma-ray results for steady sources are described in ref.[17. Another instrument, CALET Gamma-ray Burst Monitor (CGBM) [19], is a gamma-ray burst (GRB) monitor using two different kind of scintillators $\left(\mathrm{LaBr}_{3}(\mathrm{Ce})\right.$ and $\left.\mathrm{BGO}\right)$ to achieve a broad energy coverage. Results from CGBM will be presented separately [20].

CAL uses two gamma-ray trigger modes: a low-energy gamma-ray (LEG) mode with an energy threshold $\sim 1 \mathrm{GeV}$ used at low latitudes and following a CGBM burst trigger, and a highenergy (HE) mode with a threshold $\sim 10 \mathrm{GeV}$ used in normal operation irrespective of geomagnetic latitude [17]. Around the trigger time of GW151226, between 03:30 and 03:43 UT, CAL was performing regular scientific data collection operating in LEG mode. The high voltages of CGBM were set at the nominal values around 03:20 UT and turned off around 3:40 UT to avoid high background radiation area. There was no CGBM on-board trigger at the trigger time of GW151226. In the case of GW170104, CAL was operating in the high energy trigger mode with an energy threshold of $10 \mathrm{GeV}$ at the trigger time since ISS was orbiting in the high latitude region. In this paper we report on the analysis of the CAL data for these two events. First results on the analysis of GW151226 were already reported [1], and here we describe results with a refined analysis.

\section{Analysis and results}

\subsection{CALET/CAL analysis of gamma-ray events}

We apply a gamma-ray selection by tracking pair creation events in IMC [16, 17] for the flight data. The gamma-ray event selection used in this analysis is basically the same as the one de- 
scribed in ref.[16], although a stronger cut was applied by requiring three or more hits for track reconstruction. This ensures a higher tracking quality in exchange for a reduction of 1 radiationlength in conversion materials (Tungsten) usable for pair creation in the imaging calorimeter. We require the tracks cross the CHD (full area) to the TASC-top (except for the $2 \mathrm{~cm}$ margin around the outside, see Fig 10 so that reliable reconstruction of events is possible, and apply cuts to select electromagnetic showers. Finally we select gamma-ray candidates with no hits in CHD. According to the simulation study that has generated events around the instrument isotropically, we estimate that the highest gamma-ray efficiency is achieved around $10 \mathrm{GeV}$ with an efficiency of $50 \%$ relative to a geometrical factor of $420 \mathrm{~cm}^{2} \mathrm{sr}$, which is the $100 \%$ efficiency case, by applying the event selections described above. The effective areas for various incident angles are shown in Fig 2 as a function of energy. Analysis presented here is optimized to enlarge the field-of-view as wide as possible, so we use a different geometrical cut from the one used in ref. [17 which is optimized for point source analysis. Then we also have to

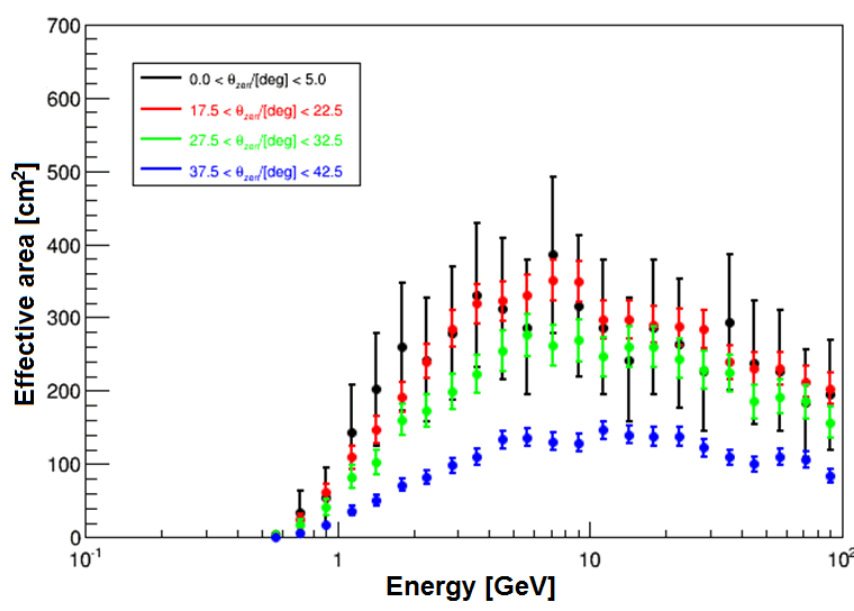

Figure 2: Effective area of CAL for gamma rays as a function of energy in the low-energy gamma-ray mode for various ranges of incident zenith angles. Error bars indicate statistical uncertainties due to Monte Carlo statistics. reject gamma-ray candidates which come through the ISS structures (such as solar panels) to remove events generated by cosmic-ray interactions with these structures, which produce event clusters clearly visible in our field-of-view. Incident gamma-ray energies were estimated based on the deposited energy in CAL considering the geometry conditions.

\subsection{Diffuse Galactic gamma-ray flux}

Our long-term CAL observation of Galactic diffuse gamma-rays in the low-energy gamma-ray mode clearly indicates the Galactic plane enhancement on the count map as shown in Fig 3 The comparison of our data and the Galactic diffuse radiation model developed by the Fermi-LAT team [21] is shown in Fig 4 as a distribution of gamma-ray events along the Galactic latitude summed over the Galactic longitude range, $-80^{\circ}<\ell<80^{\circ}$. General consistency seen in this plot shows the validity of our analysis for gamma rays observed in LEG mode of CAL. (Note that we did not subtract contribution of point sources such as the Galactic center so we do not expect perfect match here.)

\subsection{Search for a counterpart of GW151226}

A search for gamma-ray events associated with GW151226 was carried out using the CAL data in the time interval from -525 to $+211 \mathrm{~s}$ around the LIGO trigger $\left(T_{0}\right)$. The CAL was operational in low-energy gamma-ray mode with an energy threshold of $1 \mathrm{GeV}$ in this time period. We found no candidates in this time window. 


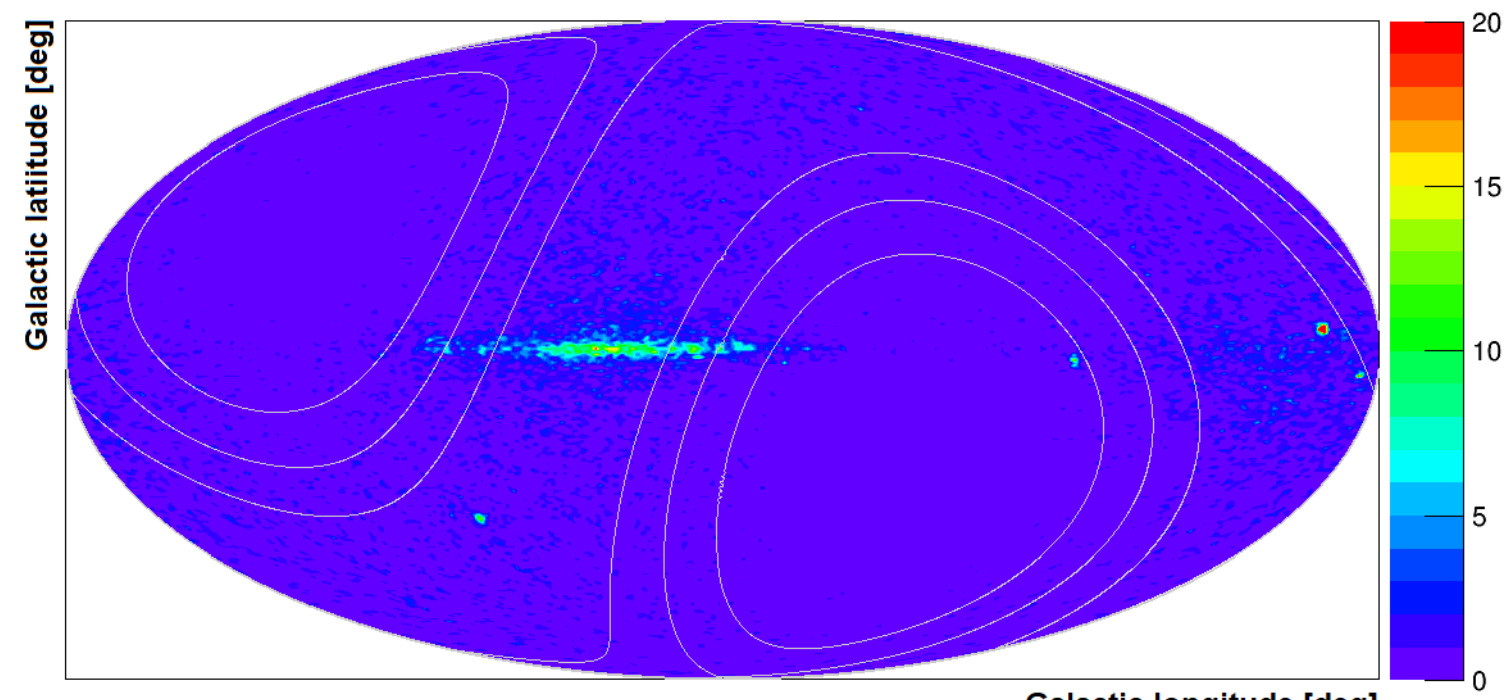

Galactic longitude [deg]

Figure 3: Count map of gamma-ray candidates obtained in the low-energy gamma-ray mode during the time period from 2015 October 13 to 2017 May 31 expressed in the Galactic coordinates. The color bar is shown in unit of counts per $2.56 \times 10^{-4} \mathrm{sr}\left(\approx 0.52^{\circ}\right.$ radius circle $)$. Also shown by contours are the exposure calculated for this period $(25,50$ and $75 \%$ of the full level). One can see some point sources clearly: see ref. [17] for discussion.

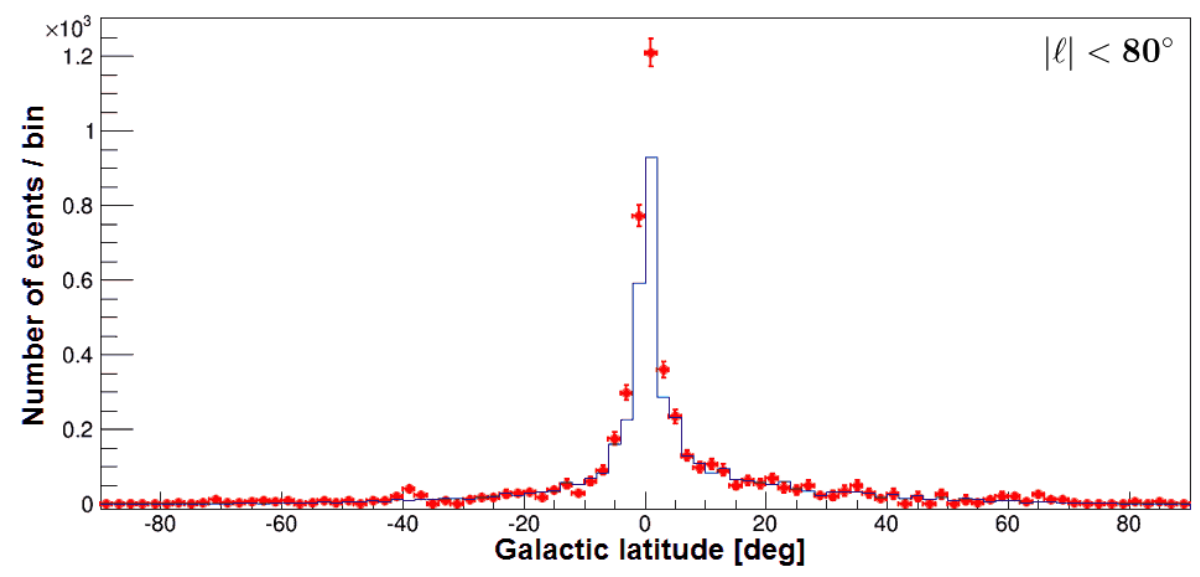

Figure 4: Galactic latitude distribution of gamma-ray candidates observed by CAL summed over the Galactic longitude range, $-80^{\circ}<\ell<80^{\circ}$, in the time period from 2015 October 13 to 2017 May 31 compared with an expectation based on the Galactic diffuse emission model (shown by a histogram) developed by the Fermi-LAT team [21].

Since the searched location for the GW151226 counterpart is significantly far from the Galactic plane, contamination of background gamma-rays is expected to be small. We calculated expected number of background events in this time window by comparison of the observed data with a prediction by the Galactic diffuse model [21]. Here we used the sky region with $|l|<80^{\circ}$ and $|b|>10^{\circ}$ to avoid the Galactic plane. Then the expected background is 0.065 events in the time window $\left[T_{0}-525 \mathrm{~s}, T_{0}+211 \mathrm{~s}\right]$ for the sky region covering $35 \%$ of the summed LIGO probabilities. This means the CAL observation is virtually background free in such a short time period. 


\subsection{Upper limit on energy flux from GW151226}

The upper limit of the CAL observation in this $736 \mathrm{~s}$ long period is estimated as follows. First, we calculated the effective area and the resultant exposure map in the time window for the 1-10 $\mathrm{GeV}$ band. At lower energies, the effective area gradually decreases below $10 \mathrm{GeV}$ and reaches zero around $500 \mathrm{MeV}$. Next, we estimated the limiting flux corresponding to 2.44 events, which is the $90 \%$ confidence limit for null observation, assuming a single power-law model with a photon index of -2 by applying the estimated exposure map. The assumed photon index of -2 is a typical photon index of the Fermi-LAT GRBs in the GeV energy range [22]. Figure 5 shows the sky map of the flux upper limit at the $90 \%$ confidence level. The estimated $90 \%$ C.L. upper limit is $1.5 \times 10^{-7} \mathrm{erg} \mathrm{cm}^{-2} \mathrm{~s}^{-1}$ in the $1-10 \mathrm{GeV}$ band where CAL reaches $15 \%$ of the integrated LIGO probability $(\sim 1.1 \mathrm{sr})$. If we increase the sky region to contain $25 \%$ of LIGO probability, the upper limit is $5.4 \times 10^{-7} \mathrm{erg} \mathrm{cm}^{-2} \mathrm{~s}^{-1}$ in the same energy band. The CAL upper limit in luminosity is estimated as $3.4(13) \times 10^{48} \mathrm{erg} \mathrm{s}^{-1}$ for a source distance of $440 \mathrm{Mpc}$ for coverage of $\sim 15(25) \%$ of LIGO probability regions. In comparison, the upper limit in the energy flux in the $0.1-1 \mathrm{GeV}$ band as reported by Fermi-LAT (assuming a single power-law spectrum with a photon index of -2) is $3 \times 10^{-10} \mathrm{erg} \mathrm{cm}^{-2} \mathrm{~s}^{-1}$ (95\% C.L.) for the time window $\left[T_{0}, T_{0}+1 \times 10^{4} \mathrm{~s}\right]$ [8], corresponding to $\sim 4 \times 10^{-9} \mathrm{erg} \mathrm{cm}^{-2} \mathrm{~s}^{-1}$ for the 736-s CALET time window.

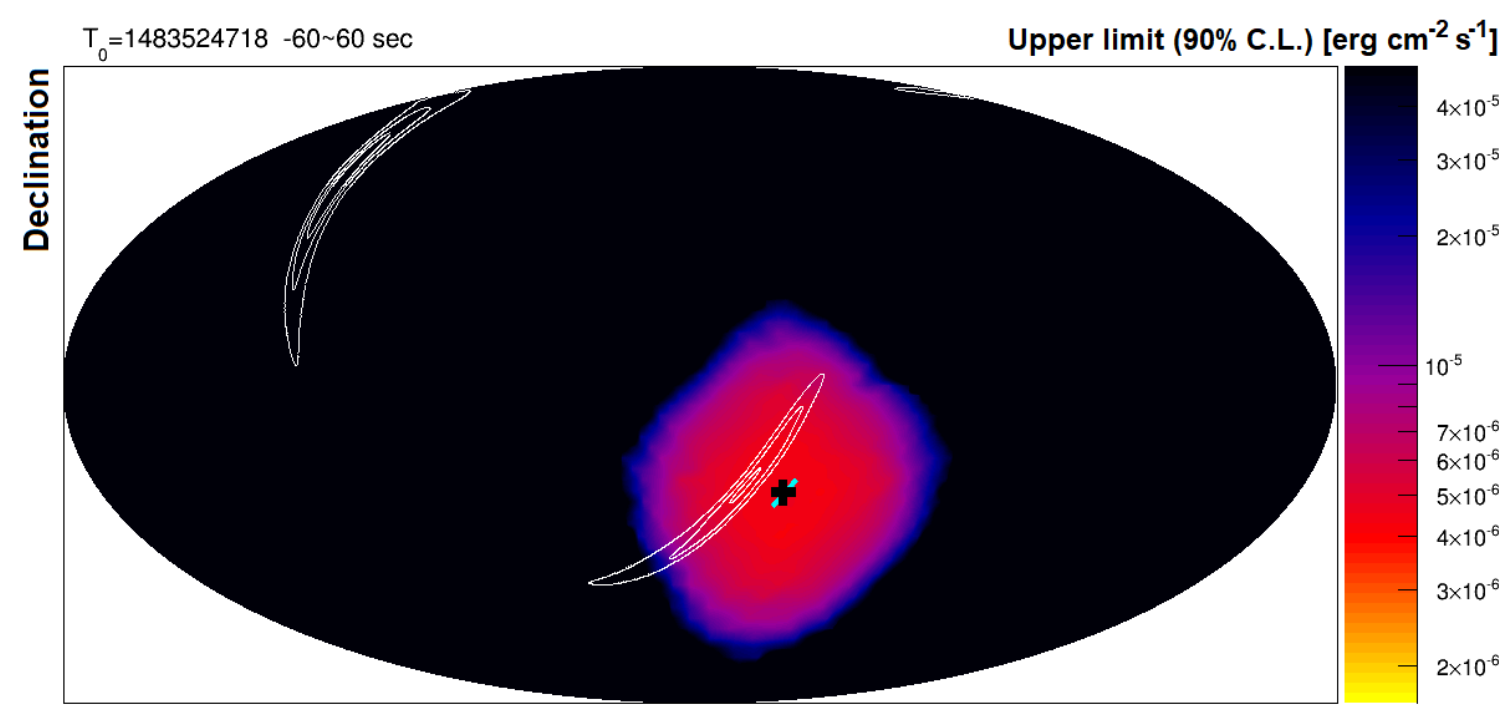

Right ascension

Figure 5: Upper limit (90\% C.L.) on energy flux in the $1-10 \mathrm{GeV}$ from GW151226 in the time window $\left[T_{0}-525 \mathrm{~s}, T_{0}+211 \mathrm{~s}\right]$ shown in the equatorial coordinates. Thick cyan line shows the locus of the fieldof-view center of CAL, and the plus symbol is that at $T_{0}$. White contours show the localization significance map of the gravitational wave signal reported by LIGO.

We also calculated upper limits on gamma-ray energy flux in smaller time windows since we have no knowledge of the time profile of the possible electromagnetic emission accompanying gravitational wave events. When we set the window as $\left[T_{0}-60 \mathrm{~s}, T_{0}+60 \mathrm{~s}\right]$, the upper limit in the $1-10 \mathrm{GeV}$ band is $1.6(4.1) \times 10^{-6} \mathrm{erg} \mathrm{cm}^{-2} \mathrm{~s}^{-1}$ for the summed LIGO probabilities inside the 
CAL field of view is $\sim 15(20) \%$. If we set the window as $\left[T_{0}-1 \mathrm{~s}, T_{0}+1 \mathrm{~s}\right]$, the upper limit in the $1-10 \mathrm{GeV}$ band is $1.0 \times 10^{-4} \mathrm{erg} \mathrm{cm}^{-2} \mathrm{~s}^{-1}$ for the summed LIGO probabilities inside the CAL field of view is $\sim 15 \%$.

\subsection{Analysis of a counterpart of GW170104}

For the time period in the vicinity of the trigger time of G268556 $\left(T_{0}\right)$ corresponding to GW170104, CAL was operating in high energy trigger mode with an energy threshold of $10 \mathrm{GeV}$. Using CAL data, we have searched for gamma-ray events above $10 \mathrm{GeV}$ from $-60 \mathrm{~s}$ to $+60 \mathrm{~s}$ from the GW trigger time and found no candidates. The number of background events in this time window is estimated to be $4.0 \times 10^{-3}$. Thus we obtained an upper limit on the gamma-ray energy flux of $8.3 \times 10^{-6} \mathrm{erg} \mathrm{cm}^{-2} \mathrm{~s}^{-1}$ at $90 \%$ C.L. in the $10-100 \mathrm{GeV}$ energy band for the sky region covering $30 \%$ of the summed LIGO probabilities (Fig 6 . If we set the narrower time window as $\left[T_{0}, T_{0}+1 \mathrm{~s}\right]$, the background estimate is $3.3 \times 10^{-5}$ and the $90 \%$ C.L. upper limit is $1.1 \times 10^{-3} \mathrm{erg} \mathrm{cm}^{-2} \mathrm{~s}^{-1}$ for the same sky region. Similarly, for the time window of $\left[T_{0}, T_{0}+10 \mathrm{~s}\right]$, the background estimate is $3.3 \times 10^{-4}$ and the $90 \%$ C.L. upper limit is $1.1 \times 10^{-4} \mathrm{erg} \mathrm{cm}^{-2} \mathrm{~s}^{-1}$.

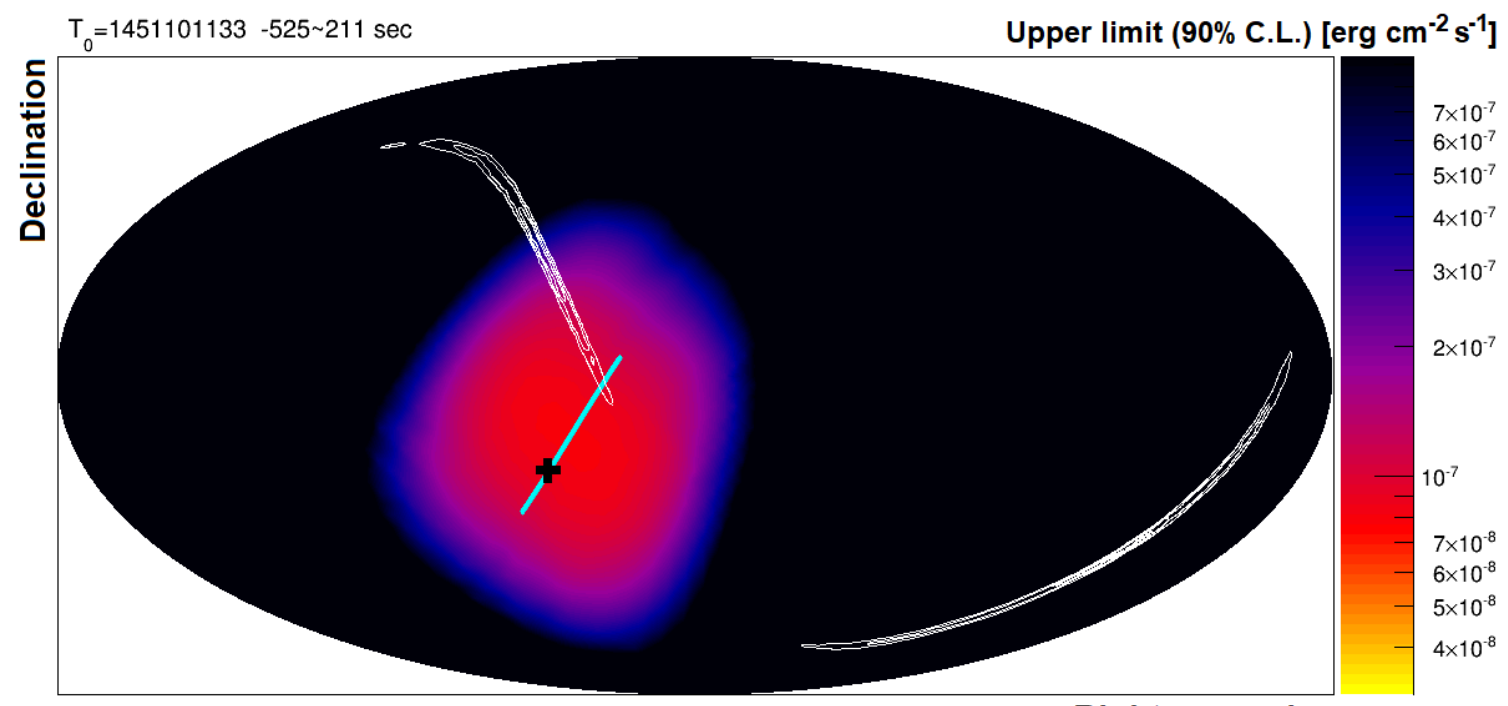

Right ascension

Figure 6: Upper limit (90\% C.L.) on energy flux in the 10-100 GeV energy band from GW170104 in the time window $\left[T_{0}-60 \mathrm{~s}, T_{0}+60 \mathrm{~s}\right]$ shown in the equatorial coordinates. Thick cyan line shows the locus of the field-of-view center of CAL, and the plus symbol is that at $T_{0}$. White contours show the localization significance map of the gravitational wave signal reported by LIGO.

\section{Discussion}

Since mergers of stellar-mass black holes (BHs), such as GW150914 observed by LIGO [1], are not expected to have electromagnetic counterparts in general, the possible gamma-ray transient source reported by Fermi-GBM [3] attracted much interest, although there is no further supporting evidence by other electromagnetic detectors. Because black hole mergers are not as rare as had 
been supposed before the LIGO discovery, there can be expected to more opportunities to observe their electromagnetic counterparts. If the evidence grows in the future, our understanding of the merger events would be forced to change drastically.

Here we report upper limits on gamma-ray energy fluxes around the LIGO trigger times of GW151226 and GW170104 for the probable sky regions estimated by gravitational wave events based on observation by CALET/CAL on ISS in the 1-10 GeV energy band and in the 10-100 $\mathrm{GeV}$ band, respectively. These results, with other electromagnetic observations and follow-ups, can provide observational constraints for theoretical models for electromagnetic counterparts to black hole mergers. As long as the CALET mission continues, we will monitor gamma-ray emission from any kind of burst-like events at the sensitivity level as described here.

Acknowledgment This work was supported in part by JSPS Grant-in-Aid for Scientific Research Grant Number JP16K05382, JP26220708, and JP17H02901.

\section{References}

[1] O. Adriani et al., Astrophys. J. Lett., 829: L20 (2016).

[2] B.P. Abbott et al., Phys. Rev. Lett. 116, 061102 (2016).

[3] V. Connaughton et al., Astrophys. J. Lett., 826: L6 (2016).

[4] V. Savchenko et al., Astrophys. J., 820: 36 (2016).

[5] B.P. Abbott et al., Astrophys. J. Lett. 826, L13 (2016).

[6] B.P. Abbott et al., Phys. Rev. Lett. 116, 241103 (2016).

[7] B.P. Abbott et al., Phys. Rev. X 6, 041015 (2016).

[8] J.L. Racusin et al., Astrophys. J. 835: 82 (2017).

[9] J. Wood et al., GCN Circ. 19156 (2016).

[10] V. Bhalerao et al., GCN Circ. 19401 (2016).

[11] B.P. Abbott et al., Phys. Rev. Lett. 118, 221101 (2017).

[12] V. Bhalerao et al., arXiv:1706.00024

[13] M. Serino et al., GCN Circ. 20507 (2017).

[14] F. Verracchia et al., arXiv:1706.00029

[15] S. Torii for the CALET Collaboration, Proc. 34th ICRC (Hague, Netherland, 2015), \#581.

[16] M. Mori for the CALET Collaboration, Proc. 33rd ICRC (Rio de Janeiro, Brazil, 2013), \#0248.

[17] N. Cannady for the CALET Collaboration, in these proceedings.

[18] Y. Asaoka et al., Astropart. Phys. 91, 1 (2017).

[19] K. Yamaoka for the CALET Collaboration, Proc. 7th Huntsville Gamma-Ray Burst Symposium (Nashville, USA, 2013), paper 41 in eConf Proceedings C1304143.

[20] K. Yamaoka for the CALET Collaboration, in these proceedings.

[21] F. Acero et al., Astrophys. J. Suppl. 223, 26 (2016).

[22] M. Ackermann et al., Astrophys. J. Suppl. 209, 11 (2013). 